\title{
Excitation pathways in resonant inelastic x-ray scattering of solids
}

\author{
Christian Vorwerk, ${ }^{1,2,{ }^{*}}$ Francesco Sottile, ${ }^{3,2}$ and Claudia Draxl ${ }^{1,2}$ \\ ${ }^{1}$ Physics Department and IRIS Adlershof, Humboldt-Universität zu Berlin, 10099 Berlin, Germany \\ ${ }^{2}$ European Theoretical Spectroscopy Facility (ETSF) \\ ${ }^{3}$ LSI, Ecole Polytechnique, CNRS, CEA, Institut Polytechnique de Paris, F-91128 Palaiseau, France
}

(Received 28 February 2020; accepted 24 August 2020; published 7 October 2020)

\begin{abstract}
Resonant inelastic x-ray scattering (RIXS) is a powerful spectroscopic technique that offers an elemental- and orbital-selective probe of the electronic excitations over a huge energy range. We present a many-body approach to determine RIXS spectra in solids, yielding an intuitive expression for the RIXS cross section in terms of pathways between intermediate many-body states containing a core hole, and final many-body states containing a valence hole. Explicit excited many-body states are obtained from the diagonalization of the Bethe-Salpeter equation in an all-electron framework. For the paradigmatic example of the fluorine $K$ edge of LiF, we show how the excitation pathways determine the spectral shape of the emission, and demonstrate the nontrivial role of electron-hole correlation in the RIXS spectra.
\end{abstract}

DOI: 10.1103/PhysRevResearch.2.042003

Resonant inelastic x-ray scattering (RIXS) spectroscopy is an important probe to unravel the nature of elementary excitations. This is owing to its unprecedented versatility in probing a wide range of elementary excitations and its ability to achieve high energy and momentum transfer. Thus, RIXS has been employed to study excitations in a wide range of crystalline materials [1-4] and molecules [5-7]. To analyze and predict the complex RIXS spectra, accurate $a b$ initio simulations are paramount. However, the inherent complexity of the microscopic RIXS process has posed a challenge for any theoretical description, as both the effects of electron-hole interactions, as well as the coherence of the RIXS process have to be included. Thus, first-principles approaches for RIXS in solids have been derived on various levels of sophistication. This started with the independent-particle approximation (IPA) within density functional theory (DFT) [8-12]. An improvement was obtained by including electronhole interactions still relying on the core-hole approximation $[13,14]$. This does not capture the full picture, because electron-hole correlation plays a crucial role in RIXS even in weakly correlated materials. As both the intermediate and the final excited state of the process contain an electronhole pair, a more accurate approach is required, as provided within many-body perturbation theory by the solution of the Bethe-Salpeter equation (BSE) [15-19]. This method is state of the art for the calculation of optical and X-ray absorption spectroscopy of condensed matter [20-26]. For selected

\footnotetext{
*vorwerk@physik.hu-berlin.de

Published by the American Physical Society under the terms of the Creative Commons Attribution 4.0 International license. Further distribution of this work must maintain attribution to the author(s) and the published article's title, journal citation, and DOI.
}

systems, alternative wave-function-based methods have been developed [27-30].

In this Rapid Communication, we propose an analytical form of a RIXS cross section in terms of coherent excitation pathways from all possible intermediate to all possible final many-body states weighted by the rates of the initial x-ray absorption. This expression provides a powerful analysis tool to infer about the origin and the making of a RIXS spectrum. We answer questions, such as what are the most important contributions, how do they interfere, why are optical features masked, and more. By offering an intuitive interpretation of the RIXS process, our formalism represents a significant step forward in the overall understanding of RIXS.

Fundamentally, the RIXS process is a second-order term of the electron-photon interaction and is commonly described in a two-step model [1,2]: In the first step, an incoming X-ray photon with energy $\omega$ is absorbed, leading to the excitation of a tightly bound core electron to the conduction band. Subsequently, a valence electron fills the core hole by emitting an $\mathrm{x}$-ray photon with smaller energy $\omega^{\prime}$. The system thus reaches a many-body state with a hole in a valence state and an excited electron in a conduction state. This final state is similar to that of optical absorption, while the selection rules of the latter and the resonant scattering process can differ significantly. Within RIXS, the absorption and emission processes occur coherently, i.e., the entire process cannot simply be considered as an absorption followed by an emission [8,31]. Rather, the final state of the absorption process determines the possible emission processes. Through this coherence, RIXS spectroscopy offers an element- and orbital-selective probe of elemental electronic excitations, because the absorption edge can be selected such as to allow for emission from specific valence states only. The double-differential cross section (DDCS) $\frac{d \sigma}{d \Omega d \omega^{\prime}}$ of scattering an X-ray photon with energy $\omega$ and polarization $\mathbf{e}_{1}$, such that an x-ray photon with energy $\omega^{\prime}$ and polarization $\mathbf{e}_{2}$ is emitted, is given by the 
Heisenberg-Kramers formula [32] as

$$
\begin{aligned}
\frac{d^{2} \sigma}{d \Omega d \omega^{\prime}} \propto & \sum_{F}\left|\sum_{I} \frac{\left\langle F\left|\hat{D}^{\dagger}\left(\mathbf{e}_{2}\right)\right| I\right\rangle\left\langle I\left|\hat{D}\left(\mathbf{e}_{1}\right)\right| 0\right\rangle}{\omega-E_{I}+i \eta_{I}}\right|^{2} \\
& \times \delta\left(E_{F}-E_{0}+\omega^{\prime}-\omega\right),
\end{aligned}
$$

where the initial absorption leads to the excitation from the many-body ground state $|0\rangle$ with energy $E_{0}$ to an intermediate state $|I\rangle$ with energy $E_{I}$. The emission of an x-ray photon leads to the deexcitation into the final state $|F\rangle$ with energy $E_{F}$. Here, $\hat{D}(\mathbf{e})$ describes the dipole transition operator for a given polarization e. As mentioned in the Introduction, the Kramers-Heisenberg formula has been tackled using the BSE approach in the pioneering works of Shirley and co-workers $[15,16]$. Conversely, we propose here a reformulation of the formula, putting into evidence the role of excitation pathways permitting a more detailed analysis.

To derive explicit expressions for the corresponding oscillator strength and the coherent emission pathway, we make use of the eigenstates $A_{c v, \lambda}$ of the BSE Hamiltonian, i.e., $H^{\mathrm{BSE}} A_{\lambda}=E_{\lambda} A_{\lambda}$. Employing $A_{c \mu, \lambda_{c}}$ and $E^{\lambda_{c}}$ of the core-level $\mathrm{BSE}$, we define the oscillator strength of core absorption $t_{\lambda_{c}}^{(1)}$ as

$$
t_{\lambda_{c}}^{(1)}=\sum_{c \mu \mathbf{k}}\left[A_{c \mu \mathbf{k}, \lambda_{c}}\right]^{*} d_{c \mu \mathbf{k}},
$$

where $c$ are the conduction states of the system, $\mu$ the core states, and $d_{c \mu \mathbf{k}}(\mathbf{e})=\mathbf{e} \cdot\langle c \mathbf{k}|\mathbf{p}| \mu \mathbf{k}\rangle$ the momentum matrix elements. With $t_{\lambda_{o}, \lambda_{c}}^{(2)}$ we define the excitation pathway from the core-level excitation $\lambda_{c}$ to the valence excitation $\lambda_{o}$ as

$$
t_{\lambda_{o}, \lambda_{c}}^{(2)}=\sum_{c v \mathbf{k}} \sum_{\mu}\left[A_{c v \mathbf{k}, \lambda_{o}}\right]^{*} d_{\mu v \mathbf{k}}^{\prime} A_{c \mu \mathbf{k}, \lambda_{c}} .
$$

This definition allows us to finally write the RIXS cross section as

$$
\begin{aligned}
\frac{d^{2} \sigma}{d \Omega d \omega^{\prime}} & \propto \operatorname{Im} \sum_{\lambda_{o}} \frac{\left|\sum_{\lambda_{c}} \frac{t_{\lambda_{o}, \lambda_{c}}^{(2)} t_{\lambda_{c}}^{(1)}}{E^{\lambda_{c}}-\omega+i \eta}\right|^{2}}{E^{\lambda_{o}}-\left(\omega-\omega^{\prime}\right)-i \eta} \\
& =\operatorname{Im} \sum_{\lambda_{o}} \frac{\left|t_{\lambda_{o}}^{(3)}(\omega)\right|^{2}}{E^{\lambda_{o}}-\left(\omega-\omega^{\prime}\right)-i \eta},
\end{aligned}
$$

where we have introduced the RIXS oscillator strength $t_{\lambda_{o}}^{(3)}(\omega)$. Further details are provided in the Supplemental Material (SM) [33].

This compact expression of the DDCS has two advantages: First, it neatly separates terms that depend on either the excitation energy $\omega$ or the energy loss $\omega-\omega^{\prime}$ from those that are independent of energy. This allows for an efficient numerical evaluation since the most-involved term $t_{\lambda_{o}, \lambda_{c}}^{(2)}$ is frequency independent. More importantly, the expression yields an intuitive interpretation of the RIXS spectra in terms of excitonic pathways as shown in Fig. 1. In essence, the rate of the initial X-ray absorption event is given by $t_{\lambda_{c}}^{(1)}$, together with the energy conservation enforced by the denominator $E^{\lambda_{c}}-\omega+i \eta$ in Eq. (4). The absorption leads to an intermediate state $\left|\lambda_{c}\right\rangle$ [37] containing a core hole, schematically shown in Fig. 1. The final RIXS spectrum is given by the rate of the

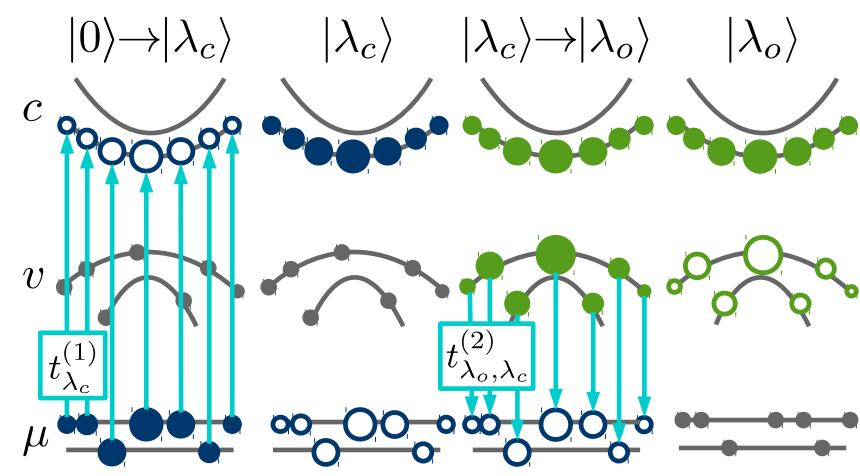

FIG. 1. Scheme of the RIXS process: A core excitation yields the intermediate many-body state $\left|\lambda_{c}\right\rangle$, where blue circles represent the distribution of the core hole (open circles) and the excited electron (solid circles). Cyan arrows indicate dipole transitions. The deexcitation from $\left|\lambda_{c}\right\rangle$ yields the final many-body state $\left|\lambda_{o}\right\rangle$. The final state is represented by green circles, where the valence hole distribution is shown in open green circles, and the distribution of the excited electron in solid ones.

first event combined with the pathway $t_{\lambda_{o}, \lambda_{c}}^{(2)}$ that connects the excited state $\left|\lambda_{c}\right\rangle$ with the final state $\left|\lambda_{o}\right\rangle$ shown in Fig. 1. The pathways $t_{\lambda_{o}, \lambda_{c}}^{(2)}$ depend strongly on the intermediate state $\left|\lambda_{c}\right\rangle$ due to the coherence of the absorption and emission processes, and the mixing between $t_{\lambda_{c}}^{(1)}$ and $t_{\lambda_{o}, \lambda_{c}}^{(2)}$ can result in destructive or constructive interference, attesting to the many-body character of such processes. Another way to look at the DDCS is provided by Eq. (5) which tells us that the overall RIXS signal is given by a combination of the energy loss and the oscillator strength $t_{\lambda_{o}}^{(3)}(\omega)$ of the whole process. The oscillator strength $t_{\lambda_{0}}^{(3)}(\omega)$ thus solely depends on the excitation energy, while the dependence on the energy loss is given by the denominator $E^{\lambda_{o}}-\left(\omega-\omega^{\prime}\right)-i \eta$.

Following the equations above, the determination of the RIXS DDCS requires the output of two BSE calculations: The first BSE calculation is performed to obtain the core excitations at a specific edge, which yields $E^{\lambda_{c}}, A_{c \mu \mathbf{k}, \lambda_{c}}$ as well as the momentum matrix elements $d_{c \mu \mathbf{k}}$ and $d_{\mu v \mathbf{k}}^{\prime}$. A second BSE calculation determines the valence excitations yielding $E^{\lambda_{o}}, A_{c v \mathbf{k}, \lambda_{o}}$. Subsequently, the absorption oscillator strength $t_{\lambda_{c}}^{(1)}$ and the pathway $t_{\lambda_{o}, \lambda_{c}}^{(2)}$ are calculated. Finally, all intermediate quantities are combined to construct the RIXS oscillator strength $t_{\lambda}^{(3)}(\omega)$ and the DDCS of Eq. (4). For a consistent treatment of the BSE eigenstates in the optical and $\mathrm{x}$-ray region, we perform the calculations using the allelectron many-body implementation in the exciting code $[38,39]$. The computational data is available in the NOMAD repository [40]. The all-electron implementation also directly yields the momentum matrix elements $d_{c \mu \mathbf{k}}$ and $d_{\mu v \mathbf{k}}^{\prime}$ between core states and conduction and valence states, respectively.

To illustrate our approach, we present in the following results for the $\mathrm{F} K$ edge of LiF. Due to its large band gap, strong effects of the electron-hole interaction are observed, as indicated by the presence of bound excitons in both the valence and the core regimes. The calculated RIXS spectra as a function of the emission energy are shown in Fig. 2 for selected excitation energies. For an excitation energy of $690.8 \mathrm{eV}$, 


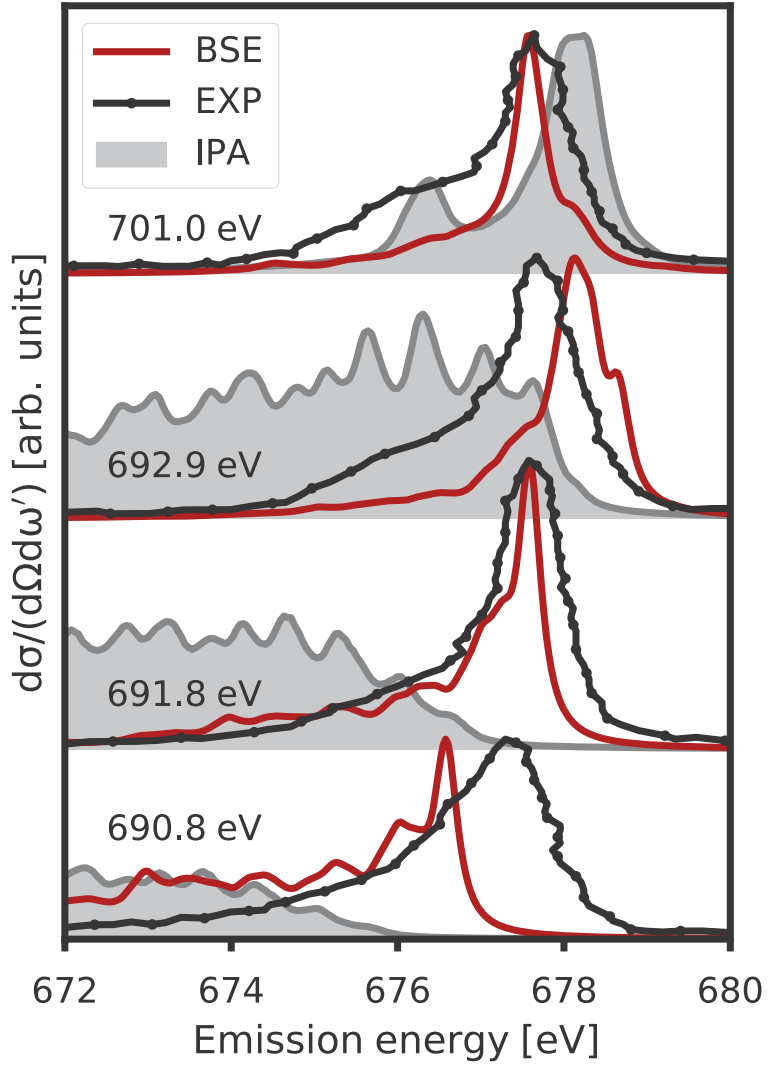

FIG. 2. Calculated (red) and experimental (black) [34] RIXS spectrum of the fluorine $K$ edge in LiF. Both spectra are normalized for each absorption energy. A Lorentzian broadening of $0.15 \mathrm{eV}$ is employed in the calculated spectra.

below the absorption onset of approximately $691.8 \mathrm{eV}$, the calculated spectrum has a peak at $676.8 \mathrm{eV}$, which slowly decays at lower emission energies, i.e., the maximum of the scattering occurs at a loss of $14 \mathrm{eV}$, with considerable contributions at a higher-energy loss. With increasing excitation energy, the peak becomes narrower and moves to a slightly higher emission energy. The broad feature at the lower emission energy is strongly suppressed for excitations of approximately $691.8 \mathrm{eV}$, while a shoulder in the emission appears for even higher excitations. The calculated spectra at a given excitation energy, as well as the change as a function of the excitation energy, are in good agreement with their experimental counterparts [34]. The main difference between the calculated and experimental spectra occurs for excitation energies below the absorption onset, where the RIXS signal is weak. The discrepancy is partly due to stray-light contributions in the experimental spectra, which are discussed in the SM [33] in more detail.

Our approach allows for a deeper analysis of the RIXS spectra, the results of which are shown in Fig. 3. For excitation energies below the absorption onset of the core edge, i.e., at approximately $691.8 \mathrm{eV}$, the cross section is small, since the F $1 s$ states are not excited resonantly. This case is discussed in the SM [33]. When the excitation energy is in resonance with the absorption onset, the spectrum changes abruptly. The oscillator strength increases tremendously and is shifted to a distinct loss peak at $14.6 \mathrm{eV}$. For higher absorption energies, this peak shows a linear dispersion. It corresponds to the strong emission observed for absorption energies of 691.8, 692.8, and $701 \mathrm{eV}$ in Fig. 2. Furthermore, this feature loses oscillator strength and widens with increasing excitation energy, thus introducing a shoulder at a higher loss with increasing relative intensity.

As the shape of the RIXS spectrum is determined by the excitation pathways, we now have a closer look at the $t^{(2)}$ matrix. The top right of Fig. 3 shows this matrix for the first 500 core and 1000 valence excitations, which determine the RIXS cross sections for excitation energies between 680.1 and $696.7 \mathrm{eV}$ and energy losses between 12.8 and $18.3 \mathrm{eV}$. It shows a pronounced band-matrix form, i.e., the largest contributions are observed along the diagonal. From Eq. (3), two factors can be inferred that lead to large pathway matrix elements. First, the transition from the valence hole distribution of the final state to the core hole has to be dipole allowed, and second, the distributions of the excited electron of the intermediate and final state have to be similar. For core excitations with increasing energy, the excited electron is distributed farther from the band gap, and the same holds true for optical excitations with increasing energy. This similarity leads to the band-matrix form of $t^{(2)}$. Moreover, we find that for core excitations at higher energies, pathways to more and more valence excitations are possible, and therefore the shoulder at higher loss is getting more pronounced.

Although the elements of $t^{(2)}$ yield insight into the origin of the features in the RIXS spectrum, they do not solely define it. While the pathway between the lowest excitations in the optical and core spectrum is very strong, surprisingly, the excitonic peak that dominates the optical absorption spectrum at $12.7 \mathrm{eV}$ is not observed in the RIXS spectrum. This strongly bound exciton is formed by a complicated interplay of the bottom of the conduction band, dominated by the Li $s$ states, and the top of the valence band, formed by the F $p$ states [41]. In the corresponding RIXS spectrum, a core excitation into the Li $s$ states at the bottom of the conduction band is not possible, as $s \rightarrow s$ transitions are dipole forbidden. While the $t^{(2)}$ matrix element between the dark exciton in the $\mathrm{F} K$ edge and the bound exciton in the optical spectrum is considerable, the $t^{(1)}$ entries are zero, as the initial excitation of the dark exciton is prohibited. We stress that this kind of analysis in terms of the interplay and interference of independent-particle transitions has been made only possible by our reformulation of the Kramers-Heisenberg formula.

Finally, we demonstrate the importance of electron-hole interactions by comparing in Fig. 2 the RIXS spectra obtained by the BSE with those from the independent-particle approximation (IPA). For low excitation energies, a broad emission spectrum is predicted within the IPA, missing the pronounced peak found in both the experimental spectra and our BSE calculations. At an excitation energy of $701 \mathrm{eV}$, the agreement between the IPA and BSE spectra improves, because the effect of the electron-hole interaction decreases with increasing excitation energy. Comparing in Fig. 3 the RIXS cross sections obtained from the two calculations, one notices that the strong peak at the excitation energy of $691.8 \mathrm{eV}$ and the loss of $14.8 \mathrm{eV}$ is completely missing within the IPA. 


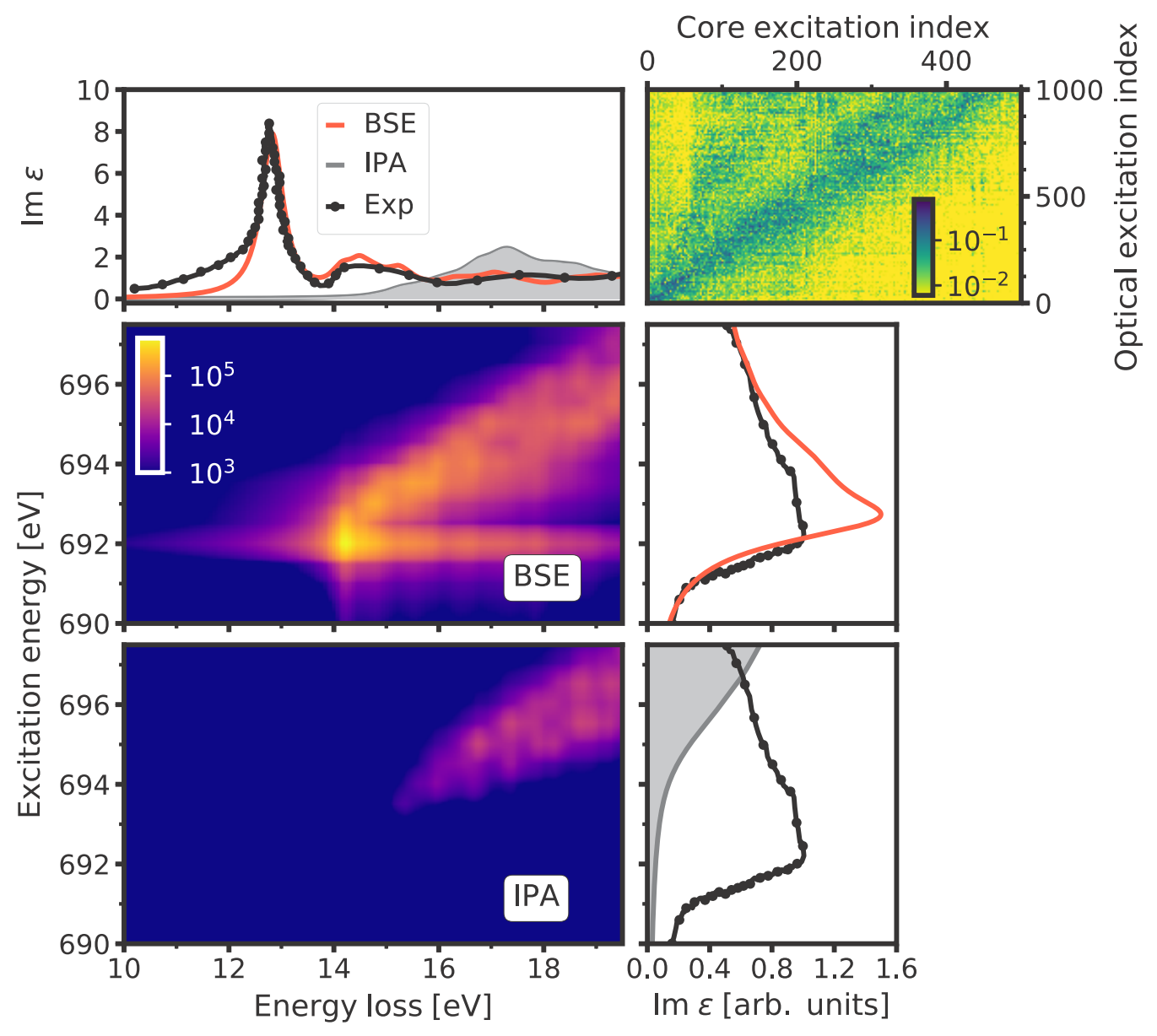

FIG. 3. F $K$ edge RIXS cross section calculated from the BSE (center top) and within the IPA (center bottom), together with the optical (top left) and the F $K$ edge (right) absorption spectrum. The calculated spectra (red BSE, gray IPA) are compared to experimental results (black) for the optical [35] and core [36] excitations. Scissor operators are applied to the calculated spectra (compare SM [33]) to correct the DFT band gap. The excitation pathways $\left|t^{(2)}\right|^{2}$ between the first 500 core and 1000 optical excitations are shown on the top right.

This comparison demonstrates that the renormalization of the RIXS spectra at low excitation energies and low-energy loss due to the electron-hole interaction is considerable, which contributes the dominant feature in the RIXS cross section. We note that in the literature, this peak has been ascribed to an excitonic peak [34]. Our first-principles approach shows that the RIXS spectrum at the core onset is more complex and requires an in-depth analysis to be unraveled.

In conclusion, we have presented in this Rapid Communication a many-body expression for the RIXS cross section in condensed-matter systems. Our all-electron full-potential approach to RIXS treats the electron-hole interaction in both core and valence excitations consistently. This is paramount to accurately predict the RIXS spectra, as we demonstrate with the example of the wide-gap insulator LiF. Equally important, our approach provides a powerful comprehensive analysis tool that allows one to trace spectral features back to the coherent pathways between the core and valence excitations as we demonstrate on the example of $\mathrm{LiF}$. $\mathrm{LiF}$ is a paradigmatic example as it contains already all the challenges typical of a RIXS spectrum, i.e., strong excitonic effects, interferences (both constructive and distructive), interplay of interband transitions with excitons and plasmons, and sensitivity to stray light. Our methodology embraces all these challenges, provides detailed and sound explanations, and promises an accurate analysis of the present and a prediction of future RIXS spectra for a wide range of materials. As a perspective, we emphasize that the approach is very general, relying on the many-body perturbation theory framework for the description of the valence and core excitations. Any improvement of the framework automatically profits the reformulation we have proposed in this Rapid Communication. At the same time, we expect more high-resolution, low-temperature experiments in the near future, as another generation of synchrotron radiation facilities is becoming available, which will enable the application of RIXS to a large variety of systems.

F.S. thanks the French Agence Nationale de la Recherche (ANR) for financial support (Grant No. ANR-19-CE30-0011). This work was performed in the framework of GraFOx, a Leibniz-ScienceCampus partially funded by the Leibniz association. C.V. acknowledges support by the Actions of the European Cooperation in Science and Technology (COST) in the COST Action MP1306 and CA17126. 
[1] A. Kotani and S. Shin, Rev. Mod. Phys. 73, 203 (2001).

[2] L. J. P. Ament, M. van Veenendaal, T. P. Devereaux, J. P. Hill, and J. van den Brink, Rev. Mod. Phys. 83, 705 (2011).

[3] G. Ghiringhelli, M. Matsubara, C. Dallera, F. Fracassi, R. Gusmeroli, A. Piazzalunga, A. Tagliaferri, N. B. Brookes, A. Kotani, and L. Braicovich, J. Phys.: Condens. Matter 17, 5397 (2005).

[4] R.-P. Wang et al., J. Phys. Chem. C 121, 24919 (2017).

[5] A. Cesar et al., J. Chem. Phys. 106, 3439 (1997).

[6] F. Hennies et al., Phys. Rev. A 76, 032505 (2007).

[7] I. Josefsson et al., J. Phys. Chem. Lett. 3, 3565 (2012).

[8] Y. Ma et al., Phys. Rev. Lett. 69, 2598 (1992).

[9] P. D. Johnson and Y. Ma, Phys. Rev. B 49, 5024 (1994).

[10] J. J. Jia, T. A. Callcott, E. L. Shirley, J. A. Carlisle, L. J. Terminello, A. Asfaw, D. L. Ederer, F. J. Himpsel, and R. C. C. Perera, Phys. Rev. Lett. 76, 4054 (1996).

[11] V. N. Strocov et al., Phys. Status Solidi B 241, R27 (2004).

[12] V. N. Strocov, T. Schmitt, J.-E. Rubensson, P. Blaha, T. Paskova, and P. O. Nilsson, Phys. Rev. B 72, 085221 (2005).

[13] M. Magnuson, M. Mattesini, C. Höglund, J. Birch, and L. Hultman, Phys. Rev. B 81, 085125 (2010).

[14] J. J. Kas, J. J. Rehr, J. A. Soininen, and P. Glatzel, Phys. Rev. B 83, 235114 (2011).

[15] E. L. Shirley, Phys. Rev. Lett. 80, 794 (1998).

[16] E. L. Shirley, J. Phys. Chem. Solids 61, 445 (2000).

[17] J. Vinson, T. Jach, M. Müller, R. Unterumsberger, and B. Beckhoff, Phys. Rev. B 94, 035163 (2016).

[18] J. Vinson, T. Jach, M. Müller, R. Unterumsberger, and B. Beckhoff, Phys. Rev. B 96, 205116 (2017).

[19] A. Geondzhian and K. Gilmore, Phys. Rev. B 98, 214305 (2018).

[20] L. Hedin, Phys. Rev. 139, A796 (1965).

[21] M. S. Hybertsen and S. G. Louie, Phys. Rev. Lett. 55, 1418 (1985).
[22] G. Strinati, Riv. Nuovo Cimento 11, 1 (1988).

[23] G. Onida, L. Reining, R. W. Godby, R. Del Sole, and W. Andreoni, Phys. Rev. Lett. 75, 818 (1995).

[24] S. Albrecht, G. Onida, and L. Reining, Phys. Rev. B 55, 10278 (1997).

[25] L. X. Benedict, E. L. Shirley, and R. B. Bohn, Phys. Rev. Lett. 80, 4514 (1998).

[26] M. Rohlfing and S. G. Louie, Phys. Rev. Lett. 81, 2312 (1998).

[27] K. Kunnus et al., J. Phys. Chem. B 117, 16512 (2013).

[28] D. Maganas et al., J. Phys. Chem. C 118, 20163 (2014).

[29] N. A. Bogdanov et al., J. Phys.: Condens. Matter 29, 035502 (2016).

[30] M. Guo et al., J. Phys. Chem. A 120, 5848 (2016).

[31] Y. Ma, Phys. Rev. B 49, 5799 (1994).

[32] H. A. Kramers and W. Heisenberg, Z. Phys. 31, 681 (1925).

[33] See Supplemental Material at http://link.aps.org/supplemental/ 10.1103/PhysRevResearch.2.042003 for a discussion of the RIXS below the absorption onset, a more extensive derivation of our expression for the cross section, and an overview of the numerical parameters for our calculations.

[34] A. Kikas, T. Käämbre, A. Saar, K. Kooser, E. Nõmmiste, I. Martinson, V. Kimberg, S. Polyutov, and F. Gel'mukhanov, Phys. Rev. B 70, 085102 (2004).

[35] K. K. Rao, T. J. Moravec, J. C. Rife, and R. N. Dexter, Phys. Rev. B 12, 5937 (1975).

[36] Y. Joly, C. Cavallari, S. A. Guda, and C. J. Sahle, J. Chem. Theory. Comput. 13, 2172 (2017).

[37] Within the Tamm-Dancoff approximation, the excited state is given as $|\lambda\rangle=\sum_{c \mu \mathbf{k}} A_{c \mu \mathbf{k}, \lambda} \hat{c}_{c \mathbf{k}}^{\dagger} \hat{c}_{\mu \mathbf{k}}|0\rangle$.

[38] C. Vorwerk, B. Aurich, C. Cocchi, and C. Draxl, Electron. Struct. 1, 037001 (2019).

[39] A. Gulans et al., J. Phys.: Condens Matter. 26, 363202 (2014).

[40] C. Vorwerk and C. Draxl, NOMAD Dataset of Calculations for LiF (2020), doi: 10.17172/NOMAD/2020.07.14-2.

[41] M. Gatti and F. Sottile, Phys. Rev. B 88, 155113 (2013). 